\title{
Purification and Characterization of Proteolytic Enzymes of Leishmania mexicana mexicana Amastigotes and Promastigotes
}

\author{
By M. F. PUPKIS* AND G. H. COOMBS \\ Department of Zoology, University of Glasgow, Glasgow G12 8QQ, UK
}

(Received 30 December 1983; revised 11 May 1984)

\begin{abstract}
Leishmania mexicana mexicana amastigote and promastigote soluble proteinases were purified using gel filtration and ion exchange chromatography. For the amastigotes, two main proteinase activity peaks were separated with both methods. These accounted for approximately $10 \%$ and $90 \%$ of the total activity. Characterization of the two activities for substrate specificity and sensitivity to inhibitors indicated that the major peak from both column methods contained enzymes with the characteristics of cysteine proteinases. SDS-polyacrylamide gel electrophoresis of the enzyme from the major peak purified by gel filtration revealed one polypeptide with a molecular weight in the region of 31000 . In contrast, the activity of the minor peak eluted from the columns was of higher molecular weight (67000) and was similar to metalloproteinases. Purification of the soluble proteinases in the promastigote of $L$. m. mexicana produced only one activity peak from both column techniques. This activity (mol. wt 67000) corresponded to the high molecular weight proteinase of the amastigote. The purified proteinases were active on 4nitroanilide and 7-amino-4-methylcoumarin derivatives of various small peptides. The high molecular weight proteinases of both amastigotes and promastigotes were similarly active against most of the peptides, suggesting a low specificity of the enzymes. In contrast, the low molecular weight amastigote proteinases were particularly active against two of the substrates, namely BZ-Pro-Phe-Arg-Nan and Z-Phe-Arg-MCA. These results indicate that a highly active, substrate-specific, soluble proteinase, with characteristics of a cysteine proteinase, is produced upon transformation of the $L$. m. mexicana promastigote to amastigote. The discovery and characterization of this enzyme offers opportunities for the development of new antileishmanial agents.
\end{abstract}

\section{INTRODUCTION}

Proteinases occur widely throughout the animal kingdom and are intimately involved in many cellular processes (North, 1982). Nevertheless, the proteolytic activities in Leishmania species, many of which are important human parasites, have been little studied. Proteinases have been reported to be present in the cultured promastigotes of several species (Camargo et al., 1978; Steiger et al., 1979; Fong \& Chang, 1981; Simon \& Mukkada, 1983). Only with Leishmania mexicana mexicana, however, have there been studies of the proteinases in the amastigotes, the stage parasitic in mammals including man (North \& Coombs, 1981; Coombs, 1982). With this species, it was found that the proteinase activity in the amastigote was considerably greater than that in the promastigote. The major amastigote proteinases were shown to be sensitive to

Abbreviations: Nan, 4-nitroanilide; MCA, 7-amino-4-methylcoumarin; PMSF, phenylmethylsulphonyl fluoride; PCMB, p-chloromercuribenzoate; DIFP, diisofluorophosphate; TLCK, $N$ - $\alpha$-tosyl-L-lysine chloromethylketone; TPCK, L-tosylamido-2-phenylethyl chloromethylketone; AZCase, azocaseinase (enzyme that catalyses AZC hydrolysis); HPAase, hide powder azurase (enzyme that catalyses HPA hydrolysis); HMW, high molecular weight; LMW, low molecular weight. 
inhibitors of cysteine proteinases, some of which were observed to be potently leishmanicidal (Coombs et al., 1982; Coombs \& Baxter 1984). It was proposed that these enzymes may play a key part in the survival of the amastigote within the host cell macrophage (Coombs, 1982).

In order to elucidate the precise role of the proteinases in the Leishmania-macrophage interaction, and to be able to exploit fully the parasite proteinases as targets for chemotherapeutic attack, it was essential first to characterize the enzymes, in particular with respect to their specificity of action. This we have carried out for the purified soluble proteinases from $L$. m. mexicana amastigotes and promastigotes; the results are presented in this papersome of the results have been reported in brief previously (Pupkis \& Coombs, 1983).

\section{METHODS}

Isolation, cultication and fractionation of cells. Leishmania mexicana mexicana amastigotes were grown in female NIH mice (Hacking \& Churchill, Huntingdon, Cambridge, UK) or in female CBA mice (Department of Zoology, University of Glasgow, Glasgow, UK) and isolated as described previously (Hart et al., 1981). The purified amastigotes were viable and essentially free from contamination by intact host cells (less than $0.2 \%$ ) and cellular debris. Promastigotes of $L$. m. mexicana were grown as described previously (North \& Coombs, 1981) and only cells which had been sub-passaged less than ten times were used in these studies. Crude homogenates of washed cells in $0.25 \mathrm{M}$-sucrose were obtained by the addition of Triton $\mathrm{X}-100$ to $0.1 \%(\mathrm{v} / \mathrm{v})$ except before chromatographic purification when homogenates in column buffer were obtained by three cycles of freezing $\left(-170^{\circ} \mathrm{C}\right)$ and thawing $\left(25^{\circ} \mathrm{C}\right)$. No significant differences in proteinases specific activities were observed between fresh samples and those stored at $-70^{\circ} \mathrm{C}$ for several weeks.

Chromatographic fractionation of proteolytic activities. All procedures were carried out at $4{ }^{\circ} \mathrm{C}$. The high speed $\left(240000 \mathrm{~g}\right.$ ) supernatant fraction from either amastigote or promastigote homogenates (from $4 \times 10^{10}$ parasites in $10 \mathrm{ml}$ ) was applied to the column in each case. The $A_{280}$ of the eluent was monitored to detect the protein peaks and the proteolytic activity of the fractions was measured using azocasein as substrate. Fractions containing the highest proteinase activities were pooled and stored at $-70^{\circ} \mathrm{C}$. Peak B and peak II activities were stored in the presence of $1 \mathrm{~mm}$-DTT. Under these conditions there was no significant loss of activity over several months.

(i) Gel filtration. A column $(2.6 \times 70 \mathrm{~cm})$ of Sephadex G-75 superfine was equilibrated in $0.15 \mathrm{M}$-phosphate buffer, $\mathrm{pH} 6 \cdot 0$, prior to sample loading. The flow rate $\left(20 \mathrm{ml} \mathrm{h}^{-1}\right)$ was maintained using a peristaltic pump and $5 \mathrm{ml}$ fractions of the eluent were collected.

(ii) lon exchange chromatography. A column $(1.6 \times 40 \mathrm{~cm})$ of DEAE-cellulose (DE-52) was equilibrated in $0.025 \mathrm{M}$-phosphate buffer, $\mathrm{pH} 6.0$, prior to sample loading. After extensive washing with the column buffer to remove unbound protein, the proteinase activities were eluted with a linear gradient of phosphate buffer $(\mathrm{pH} 6 \cdot 0)$ ranging from $0.2 \mathrm{M}$ to $1.0 \mathrm{M}$. The flow rate $\left(100 \mathrm{ml} \mathrm{h}^{-1}\right)$ was maintained using a peristaltic pump and $1 \mathrm{ml}$ fractions were collected.

Assay of proteolytic actitities. Proteinase activity was assessed using azocasein as substrate (AZCase) as described previously (Coombs, 1982). One unit (U) of AZCase activity was defined as that which caused the hydrolysis of $1 \mathrm{mg}$ azocasein $\min ^{-1}$ under the specified assay conditions. The $A{ }_{36}^{1 \%}$ of azocasein was taken as 32 .

The activities against hide powder azure and $\alpha$-casein were determined using the methodology described previously for the azocasein assay but with saturated suspensions of hide powder azure $\left(10 \mathrm{mg} \mathrm{ml}^{-1}\right.$, sonicated for $5 \mathrm{~min}$ in 10-s bursts with 10-s cooling periods at $4 \mu \mathrm{m}$ amplitude using an MSE Soniprep 150 ultransonic disintegrator) and $\alpha$-casein $\left(5 \mathrm{mg} \mathrm{ml}^{-1}\right)$ in place of the azocasein. The absorbance of the trichloroacetic acid soluble peptides, released from the substrates during the incubation period, was measured at $595 \mathrm{~nm}$ for hide powder azure at $280 \mathrm{~nm}$ for $\alpha$-casein. One unit (U) of activity was defined as that which caused the hydrolysis of $1 \mu \mathrm{g}$ protein $\min ^{-1}$ under the specified assay conditions. The $A{ }_{595}^{1 \%}$ of hide powder azure was taken as 4.4 and the $A_{280}^{1 \%}$ of $\alpha$-casein as $7 \cdot 4$.

The $\mathrm{pH}$ optima were determined for the amastigote and promastigote homogenate proteinase activities using the following buffer systems at a final concentration of $0 \cdot 15 \mathrm{M}$ : $\mathrm{pH} 4 \cdot 0-6 \cdot 0$, succinic acid/ $\mathrm{NaOH} ; \mathrm{pH} 6 \cdot 0-7 \cdot 5$, $\mathrm{NaH}_{2} \mathrm{PO}_{4} / \mathrm{Na}_{2} \mathrm{HPO}_{4} ; \mathrm{pH} 7 \cdot 5-9 \cdot 0$, Tris/HCl; and $\mathrm{pH} 9 \cdot 0-10 \cdot 0$, glycine $/ \mathrm{NaOH}$.

Peptidase activities were assayed in $0.15 \mathrm{M}$-imidazole buffer $(\mathrm{pH} 7.0)$ using 4-nitroanilide ( $\mathrm{Nan}$ ) derivatives of various peptides at a final concentration of $1.0 \mathrm{~mm}$. Stock solutions of the substrates were prepared in water and heated (to $90^{\circ} \mathrm{C}$ for $5 \mathrm{~min}$ ) to dissolve. The reaction mixtures $\left(1.5 \mathrm{ml}\right.$ total volume) were preincubated at $32^{\circ} \mathrm{C}$ for $10 \mathrm{~min}$ and the reaction was initiated by the addition of the enzyme. The change in $A_{405}$ was monitored continuously with a Pye Unicam SP 8000 spectrophotometer which was temperature controlled at $32^{\circ} \mathrm{C}$. One unit (U) of activity was defined as that which caused the release of $1 \mathrm{nmol} 4$-nitroanilide $\min ^{-1}$ under the conditions specified. The molar absorption coefficient of 4-nitroanilide was taken as $95001 \mathrm{~mol}^{-1} \mathrm{~cm}^{-1}$.

Peptidase activities were also determined using amide derivatives of 7-amino-4-methylcoumarin (MCA) in a modified assay system described previously (Morita et al., 1977). Assay mixtures consisted of $0.15 \mathrm{M}$-imidazole 
buffer $(\mathrm{pH} \mathrm{7.0)}$, enzyme $(50 \mu \mathrm{l})$ and substrate $(150 \mu \mathrm{l})$ in a total volume of $2.5 \mathrm{ml}$. Each substrate was first dissolved in DMSO and the solution was diluted in assay buffer to give a substrate concentration of $0.1 \mathrm{mM}$ in the appropriate assay mixture. The reaction mixtures were preincubated at $32^{\circ} \mathrm{C}$ for $10 \mathrm{~min}$ and the reaction was initiated by the addition of the enzyme. The fluorescence of the released MCA was monitored continuously using an MSE Spectroplus equipped with an external recorder. The measurements were carried out with excitation and emission wavelengths of $380 \mathrm{~nm}$ and $460 \mathrm{~nm}$, respectively. The instrument was standardized such that a $5 \mu \mathrm{M}$ solution of MCA in $0.1 \%(\mathrm{v} / \mathrm{v})$ DMSO gave 1.0 relative fluorescent unit. Protein concentrations were estimated by the Lowry method, using bovine serum albumin as standard.

Measurement of the effect of inhibitors on proteinases. Stock solutions of each of the inhibitors were prepared by dissolving in water, absolute ethanol or DMSO. Appropriate controls were carried out using the solvents and their effects on the enzyme were taken into account. For the azocasein assay, inhibitors at three times the final concentrations were preincubated with the enzyme for $1 \mathrm{~h}$ at $18^{\circ} \mathrm{C}$ before the addition of the substrate. For the peptidase assays (Nan and MCA substrates), inhibitors were preincubated with the enzyme for 15 min at $32^{\circ} \mathrm{C}$ prior to the addition of the substrate. Proteinases are usually characterized according to their sensitivity to a range of inhibitors. The most important inhibitors of the four types of enzymes recognized are: aspartic proteinases, pepstatin A; metalloproteinases, EDTA, EGTA and 1,10-phenanthroline; serine proteinases, PMSF and DIFP; cysteine proteinases, iodoacetic acid and PCMB. Leupeptin, antipain, TLCK and TPCK inhibit many cysteine proteinases but also some serine proteinases (North, 1982).

Polyacrylamide gel electrophoresis. Flat bed gel electrophoresis, using the discontinuous buffer system of Laemmli (1970), was used to analyse the protein banding patterns of the purified enzyme preparations. Electrophoresis was carried out in the presence of $0 \cdot 1 \%(w / v)$ SDS using the apparatus of Amos (1976). The gel dimension was $7 \times 7 \times 0.15 \mathrm{~cm}$ with the capacity of seven tracks for sample loading. An electrophoresis calibration kit of the low molecular weight range $(94000-14400)$ was used to standardize the gel system. The percentages of acrylamide used for the separation and stacker gels were 7.5 and 5 , respectively.

Materials. DEAE-cellulose (DE-52) was obtained from Whatman. Sephadex G-75 (superfine) and low molecular weight calibration kits for both column chromatography and gel electrophoresis were obtained from Pharmacia Fine Chemicals. Azocasein, $\alpha$-casein, hide powder azure, $N, N^{\prime}$-methylenebisacrylamide, acrylamide, phenylmethylsulphonyl fluoride, $p$-chloromercuribenzoate, $N$ - $\alpha$-p-tosyl-L-lysine chloromethylketone, L-1-tosylamide-2-phenylethyl chloromethylketone, DTT, iodoacetic acid, pepstatin $\mathrm{A}, N, N, N^{2}, N^{2}$-tetramethylethylenediamine, SDS, bovine serum albumin (fraction V), Coomassie brilliant blue $R$, Triton X-100, $\alpha$-cellulose, Sigmacell (type 50), 4-nitroaniline, BZ-Arg-Nan, BZ-Val-Gly-Arg-Nan, BZ-Pro-Val-Arg-Nan, BZ-Pro-Phe-Arg-Nan and TOS-Gly-Phe-Arg-Nan were obtained from Sigma. 7-Amino-4-methylcoumarin, SUC-Ala-Pro-Ala-MCA, SUCGly-Pro-Leu-Gly-Pro-MCA, BOC-Val-Pro-Arg-MCA, Z-Phe-Arg-MCA, BOC-Gin-Arg-Arg-MCA and BOCVal-Leu-Lys-MCA were obtained from Peninsula Laboratories Inc., Belmont, Calif., USA. Antipain and leupeptin were obtained from the Protein Research Foundation, Osaka, Japan. Other reagents were of Analar grade and obtained from $\mathrm{BDH}$.

\section{RESULTS}

\section{Total proteinase activity of amastigotes and promastigotes}

The AZCase activities of homogenates of L. m. mexicana amastigotes and promastigotes at a range of $\mathrm{pH}$ values are shown in Fig. 1. Whereas the amastigote activity had a sharp otpimum at $\mathrm{pH} 5 \cdot 5$, with the promastigote there was equal activity over a broad range of $\mathrm{pH}(6 \cdot 0-7 \cdot 5)$. The results from similar studies using other substrates are presented in Table 1 . With each of the three protein substrates employed, the proteinase specific activities were found to be much greater for amastigotes than for promastigotes. In each case, the amastigote activity was more than doubled by the addition of 1.0 mM-DTT, a compound with stimulates cysteine proteinases, and with each substrate the $\mathrm{pH}$ optimum ( $\mathrm{pH}$ of the greatest specific activity) was in the acidic region. The promastigote activity, however, was not significantly affected by DTT. During the characterization of the azocasein assay, involving the measurement of the effects of different concentrations of substrate, it was found that the promastigote required five times more azocasein (approximately $10 \mathrm{mg}$ AZC per $750 \mu \mathrm{l}$ assay mixture) than the amastigote for maximal activity to be obtained. The promastigote HPAase activity was too low, (less than $5 \mathrm{mU}$ (mg protein) $)^{-1}$ at $\mathrm{pH} 6.0$ compared with $450 \mathrm{mU}$ (mg protein $)^{-1}$ for amastigotes) to justify the determination of the $\mathrm{pH}$ optimum. With the synthetic substrate BZ-Arg-Nan, the specific activities in both forms of the parasite were very similar and with each the $\mathrm{pH}$ optimum was $8 \cdot 0$. 
Table 1. Activities of Leishmania mexicana proteinases against different substances and at different $\mathrm{pH}$

The figures given are the specific activities $\left[\mathrm{mU}(\mathrm{mg} \text { protein })^{-1}\right]$ at each $\mathrm{pH}$ where the activity was at a maximum. Amastigote activities were assessed in the presence of $1 \mathrm{mM}$-DTT.

$$
\begin{array}{lllllllll}
\mathrm{pH} \quad \ldots & 4.0 & 5.5 & 6.0 & 7.0 & 7.5 & 8.0 & 9.0 & 9.5
\end{array}
$$

Amastigotes

Azocasein

Hide powder azure

129

$x$-Casein

$513 \quad 463$

BZ-Arg-Nan*

409

489

Promastigotes

Azocasein

$\alpha$-Casein

BZ-Arg-Nan*

54

- BZ, $N$-benzoyl.

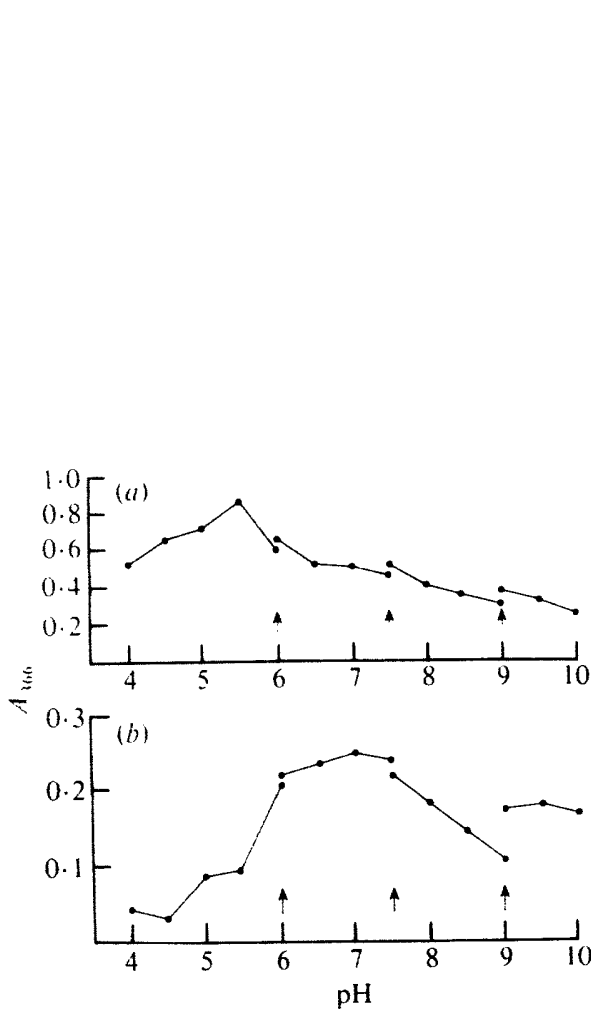

Fig. 1
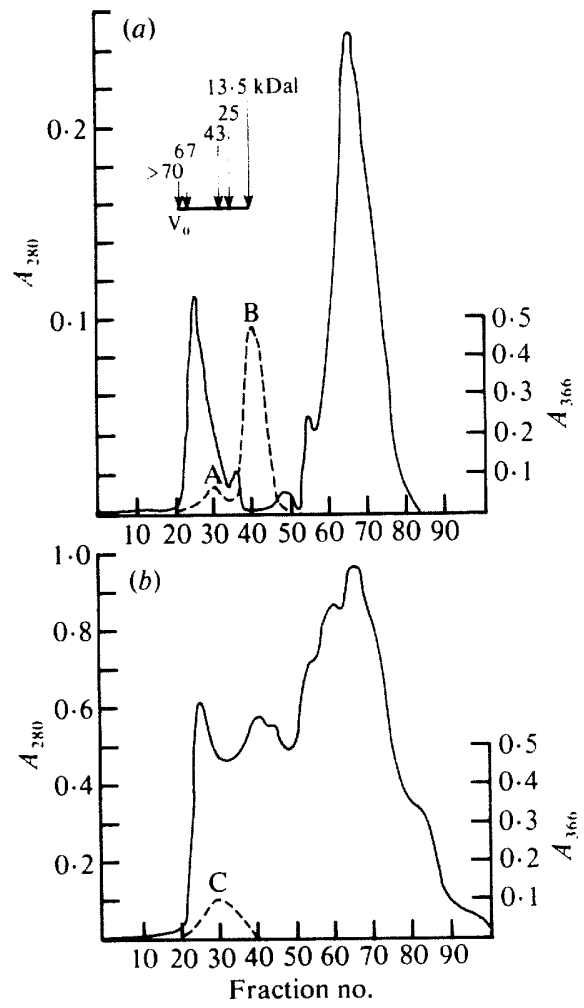

Fig. 2

Fig. 1. Effect of $\mathrm{pH}$ on the activities (measured as $A_{366}$ ) of the amastigote $(a)$ and promastigote $(b)$ proteinase, using the substrate azocasein. DTT at $1 \mathrm{~mm}$ was included only with the amastigote samples. At the $\mathrm{pH}$ values $(6 \cdot 0,7 \cdot 5$ and $9 \cdot 0)$ where the buffer systems used were changed (arrows), activities were determined with both buffers.

Fig. 2. Purification of amastigote and promastigote proteinases by column chromatography with Sephadex G-75. The high speed supernatant fraction from homogenates of either $(a)$ amastigote $(20 \mathrm{mg}$ protein) or $(b)$ promastigote $(50 \mathrm{mg}$ protein) was applied to the column. Alternate fractions were analysed for $A_{280}(-)$ and for AZCase activities (measured as $\left.A_{366} ;---\right)$. DTT at $1 \mathrm{mM}$ was included only in the assays of amastigote samples. The standards ribonuclease $A(13.5 \mathrm{kDal})$, chymotrypsinogen $A(25 \mathrm{kDal})$, ovalbumin $(43 \mathrm{kDal})$ and albumin $(67 \mathrm{kDal})$ eluted at the positions shown. $\mathrm{V}_{0}$, void volume. 
Table 2. Properties of the Leishmania mexicana proteinases purified by column chromatography with Sephadex 675

Azocasein was used at $\mathrm{pH} 6.0$ to determine the activities of the proteinases. The activities are given as a percentage of the activity in the absence of DTT and inhibitor, except for those of peak B in the presence of inhibitor, which were measured in the presence of $1 \mathrm{mM}$-DTT and are given as a percentage of the control activity with DTT. The values given are means ( \pm standard deviation) from between three and six experiments or, in three cases, from single experiments.

Percentage of total activity applied to column Specific activity [mU (mg protein) $\left.{ }^{-1}\right]$ Approx. molecular weight

\begin{tabular}{lc}
\multicolumn{1}{c}{ Addition } & Concn (mM) \\
DTT & 1.00 \\
Antipain & 0.05 \\
Leupeptin & 0.05 \\
Iodoacetic acid & 0.33 \\
TLCK & 1.00 \\
TPCK & 1.00 \\
PMSF & 0.33 \\
Pepstatin A & 0.02 \\
1,10-Phenanthroline & 1.00 \\
EDTA & 8.00
\end{tabular}

\begin{tabular}{|c|c|c|}
\hline \multicolumn{3}{|c|}{ Purified proteinases } \\
\hline Peak A & Peak B & Peak C \\
\hline $\begin{array}{c}12 \pm 4 \\
263\end{array}$ & $\begin{array}{c}88 \pm 4 \\
3026\end{array}$ & $\frac{99 \pm 2}{72}$ \\
\hline 50000 & 10000 & 50000 \\
\hline
\end{tabular}

Percentage of control activity

$\begin{array}{ccc}76 \pm 9 & 765 \pm 35 & 71 \pm 10 \\ 79 \pm 3 & 7 \pm 4 & 91 \pm 4 \\ 94 \pm 4 & 5 \pm 4 & 81 \pm 4 \\ 96 \pm 6 & 10 \pm 5 & 84 \pm 10 \\ 100 \pm 1 & 6 \pm 6 & 88 \pm 9 \\ 89 & \text { ND } & 81 \\ 95 \pm 3 & 98 \pm 3 & 93 \pm 4 \\ 97 \pm 4 & 95 \pm 5 & 100 \\ 26 \pm 22 & 100 \pm 1 & 80 \pm 29 \\ 68 \pm 24 & 96 \pm 6 & 59 \pm 18\end{array}$

ND, Not determined.

\section{Purification by gel chromatography of leishmanial proteolytic activities and their sensitivity to inhibitors}

Chromatographic purification of the soluble fraction of homogenates of the two developmental forms of the parasite on a Sephadex G-75 column (Fig. 2) revealed the presence of two distinct AZCase activity peaks for amastigotes (designated as peaks A and B) and one distinct activity peak for promastigotes (designated as peak C). With amastigotes, a 1:8 distribution ratio for the two proteinase activity peaks was observed (Table 2). Calibration of the Sephadex column, using low molecular weight standard proteins, indicated that the molecular weights of peak A and peak B activities were in the region of 50000 and 10000 , respectively. These will be referred to as the high and low molecular weight (HMW and LMW) proteinases, respectively. The LMW proteinase activity was apparently purified about 35 -fold. The HMW amastigote proteinase was inhibited by DTT, EDTA and 1,10-phenanthroline, whereas the LMW proteinase was greatly stimulated by DTT and very greatly inhibited by antipain, leupeptin, iodoacetic acid and TLCK (Table 2).

For the promastigotes, peak C contained almost $100 \%$ of the total soluble AZCase proteinase activity (Table 2). These enzymes had an apparent molecular weight of 50000 and they were purified almost 20 -fold according to the increase in the specific activity. DTT, EDTA and 1,10phenanthroline inhibited the purified promastigote enzymes; indeed the sensitivity pattern to inhibitors was very similar to that of the amastigote HMW proteinases.

The $\mathrm{pH}$ optimum of the two amastigote AZCase activity peaks was identical ( $\mathrm{pH} \mathrm{5.5)} \mathrm{to} \mathrm{that}$ of the amastigote homogenate, suggesting that no substantial changes in either the composition or the nature of the proteinases occurred during their purification.

\section{Purification by ion exchange chromatography of leishmanial proteolytic activities and their sensitivity to inhibitors}

Chromatographic purification of the amastigote and promastigote soluble proteinases on a DEAE-cellulose column revealed the presence of two major AZCase peaks for the amastigotes (designated peaks I and II) and one major AZCase activity peak for promastigotes (designated 
Table 3. Properties of the Leishmania mexicana proteinases purified by column chromatography with DEAE-cellulose

Azocasein was used at $\mathrm{pH} 6.0$ to determine the activities of the proteinases. The activities are given as a percentage of the activity in the absence of DTT and inhibitor, except for those of peak II in the presence of inhibitor, which were measured in the presence of $1 \mathrm{mM-DTT}$ and are given as a percentage of the control activity with DTT. The values given are means ( \pm standard deviation) from three or four experiments or, in two cases, from single experiments.

Percentage of total activity applied to column Specific activity [mU (mg protein) ${ }^{-1}$ ]

\begin{tabular}{lc}
\multicolumn{1}{c}{ Addition } & Concn (mM) \\
DTT & 1.00 \\
Antipain & 0.05 \\
Leupeptin & 0.05 \\
lodoacetic acid & 0.33 \\
TLCK & 1.00 \\
TPCK & 1.00 \\
PMSF & 0.33 \\
Pepstatin A & 0.02 \\
l,10-Phenanthroline & 1.00 \\
EDTA & 8.00
\end{tabular}

\begin{tabular}{ccc}
\multicolumn{3}{c}{ Purified proteinases } \\
\hline Peak I & Peak II & Peak III \\
$16 \pm 4$ & $84 \pm 4$ & $27 \pm 3$ \\
156 & 620 & 9 \\
\multicolumn{3}{c}{ Percentage of control activity } \\
\hline \multicolumn{3}{c}{. } \\
$73 \pm 8$ & $985 \pm 330$ & $57 \pm 4$ \\
$90 \pm 4$ & $8 \pm 3$ & $84 \pm 23$ \\
$90 \pm 3$ & $4 \pm 5$ & $81 \pm 27$ \\
$98 \pm 3$ & $7 \pm 1$ & $84 \pm 14$ \\
$95 \pm 7$ & $10 \pm 1$ & $82 \pm 18$ \\
73 & $10 \pm 1$ & 55 \\
$90 \pm 6$ & $100 \pm 1$ & $100 \pm 1$ \\
$95 \pm 1$ & $100 \pm 1$ & $98 \pm 3$ \\
$43 \pm 30$ & $100 \pm 1$ & $79 \pm 14$ \\
$91 \pm 1$ & $93 \pm 7$ & $97 \pm 4$
\end{tabular}

peak III). For the amastigote, a 1:6 distribution ratio for the two peaks was observed for the recovered enzyme (Table 3). The major AZCase proteinase activity (peak II) was apparently purified about sevenfold. This peak II proteinase was greatly stimulated by DTT and inhibited by antipain, leupeptin, iodoacetic acid and TLCK (Table 3). Both the amastigote peak I and the promastigote peak III AZCase activities were similarly inhibited by DTT, and 1,10phenanthroline. They also eluted from the column in the same region of the linear phosphate gradient (at a molarity of $0.4-0.5$ ), whereas the peak II amastigote proteinase eluted at a higher phosphate molarity $(0 \cdot 5-0 \cdot 6)$.

\section{Electrophoretic analysis of the purified proteinases}

The purity of the enzymes obtained from amastigotes and promastigotes by the Sephadex chromatography procedure was examined using polyacrylamide gel electrophoresis in the presence of $0 \cdot 1 \%$ SDS. In the amastigote soluble fraction and the promastigote soluble fraction many protein species were present as represented by the extent of their banding patterns (Fig. 3, tracks 1 and 4). To obtain the amastigote protein banding pattern it was necessary to lyse the cells in the presence of leupeptin $\left(100 \mu \mathrm{g} \mathrm{ml}^{-1}\right)$ to inhibit the proteinases and to prevent extensive protein degradation. The amastigote peak A preparation (track 2) contained one major staining protein (band X, mol. wt 67000) and peak B (track 3 ) only showed the presence of a fast moving band (band Y, mol. wt 31000). Use of Sephadex chromatography with molecular weight standards gives only an approximate indication of the molecular weight of the proteinases. The values obtained using SDS-gels for these enzymes should be more accurate. The promastigote peak $C$ (track 5) preparation contained an intensely stained band which had the same electrophoretic mobility as band $X$ and which was also present in tracks 1,2 and 4 . It is interesting to note that band $Y$ could not be seen in either of the two promastigote samples (tracks 4 and 5). The pattern of occurrence of bands $X$ and $Y$ in the two forms of the parasite, together with the similarity of the molecular weight values to those obtained on Sephadex and their increased staining intensity in the purified protein samples, suggests that these two protein bands corresponded to the HMW (amastigote and promastigote) and LMW (amastigote) proteinases as demonstrated by Sephadex chromatography. The gels also showed the high purity of the LMW amastigote proteinase preparation. 


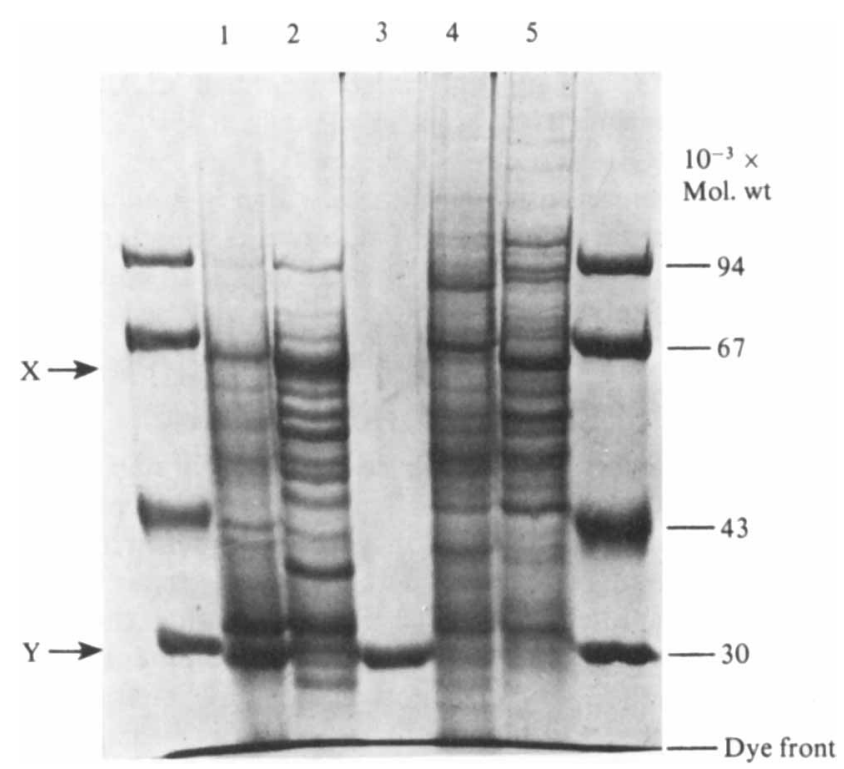

Fig. 3. Polyacrylamide gel electrophoresis of the purified leishmanial proteinases. The samples applied were: 1 , soluble fraction of amastigotes lysed in the presence of leupeptin $\left(100 \mu \mathrm{g} \mathrm{ml}^{-1}\right) ; 2$, peak $\mathrm{A} ; 3$, peak B; 4 , promastigote soluble fraction; 5, peak C. Approximately $20 \mu \mathrm{g}$ protein was loaded for the amastigote and promastigote soluble fractions and one-tenth of this quantity was present in the peak $B$ sample. The single tracks at either side of the gel contained the standard phosphorylase B ( $94 \mathrm{kDal})$, albumin $(67 \mathrm{kDal})$, ovalbumin $(43 \mathrm{kDal})$, carbonic anhydrase $(30 \mathrm{kDal})$, and at the dye front trypsin inhibitor (20.1 kDal) and lactalbumin (14.4 kDal).

Table 4. Activity of Leishmania mexicana amastigote and promastigote purified proteinases against peptide amides of 4-nitroanilide (Nan) and 7-amino-4-methylcoumarin (MCA)

The figures given are the specific activities [mU (mg protein $\left.)^{-1}\right]$ measured at $\mathrm{pH} 7 \cdot 0$.

\section{Substrate*}

BZ-Arg-Nan

BZ-Val-Gly-Arg-Nan

BZ-Pro-Val-Arg-Nan

BZ-Pro-Phe-Arg-Nan

TOS-Gly-Phe-Arg-Nan

SUC-Gly-Pro-Leu-Gly-Pro-MCA

SUC-Ala-Pro-Ala-MCA

BOC-Val-Pro-Arg-MCA Z-Phe-Arg-MCA

BOC-Gln-Arg-Arg-MCA

BOC-Val-Leu-Lys-MCA

\begin{tabular}{ccc}
\multicolumn{3}{c}{ Amastigote } \\
Homogenate & Peak A & Peak B \\
35 & 181 & 11 \\
17 & 60 & ND \\
107 & 75 & 121 \\
384 & 151 & 2259 \\
17 & 60 & 8 \\
0.61 & ND & ND \\
$0 \cdot 05$ & ND & ND \\
2 & 40 & 2 \\
72 & 156 & 3000 \\
0.57 & 11 & 4 \\
45 & 47 & 672
\end{tabular}

$\begin{array}{cc}\text { Homogenate } & \text { Peak C } \\ 25 & 180 \\ 12 & 63 \\ 15 & 31 \\ 26 & 30 \\ 12 & 63 \\ 0.2 & 1.24 \\ \text { ND } & 0.09 \\ 0.9 & 7.2 \\ 1.9 & 11.3 \\ 0.9 & 4 \\ 1.6 & 4.5\end{array}$

ND, Not detected ( $<2.0$ for Nan and $<0.01$ for MCA substrates).

* BZ, $N$-benzoyl; TOS, $N$-p-tosyl; SUC, $N$-succinyl; BOC, $t$-butyloxycarbonyl; Z, benzyloxycarbonyl.

\section{Activity of purified enzymes on 4-nitroanilide (Nan) and 7-amino-4-methylcoumarin (MCA) peptide substrates}

The proteinases were characterized using a number of chromogenic peptide derivatives of Nan and fluorogenic peptide derivatives of MCA. The HMW proteinases (peaks A and C) had similar low activities against each of the Nan derivatives of arginine tested (Table 4). However, the LMW proteinases (peak B) were very active towards the substrate BZ-Pro-Phe-Arg-Nan 
compared with the other substrates tested. The specific activity of the LMW proteinases using the substrate Z-Phe-Arg-MCA was at least three orders of magnitude greater than that measured using other substrates which had arginine as their carboxy terminal amino acid (BOCVal-Pro-Arg-MCA and BOC-GIn-Arg-Arg-MCA) and was at least five orders of magnitude greater than the activities measured using substrates which had alanine or proline instead of arginine as their carboxy terminal amino acid. These results therefore demonstrated the high specificity of the leishmanial LMW proteinases. It is interesting to note that the substrate BOCVal-Leu-Lys-MCA was a relatively good substrate for the LMW proteinases of the amastigote.

\section{DISCUSSION}

The proteinases of the amastigote of L. m. mexicana were separated into apparently the same two groups by both gel filtration and ion exchange chromatography. Likewise, the single proteinase peaks resulting from separation of promastigote extracts with the two procedures had similar properties. Gel filtration resulted in purer enzyme preparations, as shown by the appearance of only a single protein band (Y) after subjecting the low molecular weight proteinase peak to SDS gel electrophoresis. The increase in prominence of the band in the peak B preparation compared with the soluble fraction of the homogenate, which corresponded to that observed in the specific activity of the proteinase, provides strong circumstantial evidence that this band is the proteinase. The presence of band $\mathrm{Y}$ as a major component in amastigote homogenates and its apparent absence from promastigote homogenates suggests that the increase in proteinase activity upon transformation of the promastigote to amastigote is due to synthesis of the enzyme de novo. It is not possible yet to rule out an alternative suggestion, that is activation of an inactive precursor, but absence of a major band in promastigotes which has no equivalent in amastigotes makes this unlikely.

The high molecular weight proteinases which were isolated from the amastigotes by gel filtration were less pure than the low molecular weight proteinases, as demonstrated by the complexity of the protein banding pattern on SDS-gels. SDS-gel analysis of the purified proteinases demonstrated that the major protein band $X$ of the amastigote high molecular weight proteinase also occurred in the promastigote homogenate, soluble fraction and purified proteinase samples, and the band became more prominent upon purification. The purified promastigote proteinases were very similar to the amastigote high molecular weight proteinases with respect to their substrate specificity and sensitivity to inhibitors. It was concluded that these enzymes were conserved during the promastigote to amastigote transformation.

The effects of inhibitors on the purified leishmanial proteinases suggested that the high molecular weight preparations from both amastigotes and promastigotes may be mixtures of several types of enzymes. The inhibition by 1,10-phenanthroline and EDTA, however, indicates the presence of a metalloproteinase. The amastigote-specific, low molecular weight proteinases were similar to cysteine proteinases as regards their sensitivity to inhibitors and requirement for DTT.

The peptide derivatives of Nan and MCA both proved useful in studying the specificities of the leishmanial enzymes. Nan derivatives were previously used to analyse trypanosomal proteinases (North et al., 1983). The assay using MCA derivatives, developed originally for chymotrypsin (Zimmerman et al., 1976), has also proved to be suitable for other proteinases, including the blood coagulation factors, kallikreins, and urokinase (Morita et al., 1977). This fluorescent method is particularly useful because of its high sensitivity (Zimmerman et al., 1976). The use of the two methods revealed the apparent broad specificity of the purified high molecular weight proteinases, although the possibility of a mixture of enzymes means that conclusions must be tentative. Highest activity was achieved when the carboxy terminal amino acid was arginine or lysine; however, the adjacent amino acid residues had relatively little influence on the rate of hydrolysis. In contrast, the amastigote-specific, low molecular weight proteinase hydrolysed the substrates at very different rates. The results show that not only the carboxy terminal but also the adjacent two amino acid residues affected the enzyme activity. The commonly used BZ-Arg-Nan, a good substrate for the high molecular weight proteinases, 
was hydrolysed only slowly by the low molecular weight enzyme. By far the best substrates were BZ-Pro-Phe-Arg-Nan and Z-Phe-Arg-MCA. These have been reported to be suitable substrates for various mammalian proteinases, notably cathepsin B and plasma or tissue kallikreins. The amastigote low molecular weight proteinase differs from the kallikreins in several respects, including $\mathrm{pH}$ optimum and sensitivity to inhibitors. In many ways, such as sensitivity to inhibitors, the leishmanial enzyme is similar to mammalian cathepsin B. Interestingly, however, there appear to be significant differences in the substrate specificity of the two enzymes. Both BZ-Phe-Val-Arg-Nan and BZ-Gln-Arg-Arg-MCA are considered to be specific substrates for cathepsin B (Barrett \& McDonald, 1980), but neither was hydrolysed at an appreciable rate by the amastigote-specific low molecular weight proteinase.

The stage specificity of the L. m. mexicana proteinases suggests that the low molecular weight enzymes play an essential part in the survival of leishmanial amastigotes against the microbicidal activity of the host macrophage. This suggestion is supported by the discovery that inhibitors of the amastigote proteinases had potent antileishmanial activity (Coombs et al., 1982, Coombs \& Baxter, 1984). The precise role of the proteinases is still to be elucidated. It could well be, however, that end products of protein catabolism released into the parasitophorous vacuole antagonize the degradative activity of the macrophage lysosomal hydrolases indirectly by raising the $\mathrm{pH}$ of the vacuole and perhaps also through direct enzyme inhibition. The discovery of the highly active amastigote-specific proteinase of $L$. m. mexicana parasites offers opportunities for the design of new classes of anti-leishmanial drugs for which there is a great need. Cysteine proteinase inhibitors could prove to be useful leishmanicides. Studies are at present in progress to apply our knowledge concerning the specificity of the leishmanial proteinases in the development of specific inhibitors and also to exploit the amastigote proteinases by designing pro-drugs that they would activate.

We thank the MRC for financial support.

\section{REFERENCES}

Amos, W. B. (1976). An apparatus for microelectrophoresis in polyacrylamide slab gels. Analytical Biochemistry 70, 612-615.

BarRetT, A. J. \& MCDonald, J. K. (1980). Cathepsin B. In Mammalian Proteinases: Endopeptidases, pp. 267-275. Edited by A. J. Barrett \& J. K. McDonald. London: Academic Press.

Camargo, E. P., Itow, S. \& Alfieri, S. C. (1978). Proteolytic activities in cell extracts of trypanosomatids. Journal of Parasitology 64, 1120-1121.

Coombs, G. H. (1982). Proteinases of Leishmania mexicana and other flagellate protozoa. Parasitology 84, 149-155.

COOMBS, G. H. \& BAXTER, J. (1984). Inhibition of Leishmania amastigote growth by antipain and leupeptin. Annals of Tropical Medicine and Parasitology 78, 21-24.

Coombs, G. H., Hart, D. T. \& Capaldo, J. (1982). Proteinase inhibitors as antileishmanial agents. Transactions of the Royal Society of Tropical Medicine and Hygiene 76, 660-663.

Fong, D. \& Chang, K. P. (1981). Protease activity of a parasitic protozoan Leishmania mexicana. Journal of Cell Biology 91, 43A.

HaRT, D. T., Vickerman, K. \& CoOmbs, G. H. (1981). A quick, simple method for purifying Leishmania mexicana amastigotes in large numbers. Parasitology 82, 345-355.

LAEMMLI, U. K. (1970). Cleavage of structural proteins during the assembly of the head of bacteriophage T4. Nature, London 227, 680-685.
Morita, T., Kato, H., Iwanaga, S., Takada, K., Kimura, T. \& Sakakibara, S. (1977). New fluorogenic substrates of $\alpha$-thrombin, factor $\mathrm{Xa}, \mathrm{Kalli-}$ kreins and urokinase. Journal of Biochemistry 82, 1495-1498.

NORTH, M. J. (1982). Comparative biochemistry of the proteinases of eucaryotic microorganisms. Microbiological Reviews 46, 308-340.

NORTH, M. J. \& CoOmBs, G. H. (1981). Proteinases of Leishmania mexicana amastigotes and promastigotes: analysis by gel electrophoresis. Molecular and Biochemical Parasitology 3, 293-300.

NorTh, M. J., COOMbS, G. H. \& BARRY, D. (1983). A comparative study of the proteolytic enzymes of Trypanosoma brucei, $T$. equiperdum, $T$. evansi, $T$. vivas, Leishmania tarentolae and Crithidia fasciculata. Molecular and Biochemical Parasitology 9, 161-180.

PUPKIS, M. F. \& CoOMBs, G. H. (1983). Proteinases of Leishmania mexicana: purification and characterisation. Parasitology 87, xl.

Simon, M. W. \& MukKadA, A. J. (1983). Intracellular protein degradation in Leishmania tropica promastigotes. Molecular and Biochemical Parasitology 7, 1927.

Steiger, R. F., Van HoOF, F., BontemPS, J., NysSENSJADIN, M. \& DrueTZ, J. E. (1979). Acid hydrolases of trypanosomatid flagellates. Acta Tropica 36, 335341.

Zimmerman, M., Yurewicz, E. C. \& Patel, G. (1976). A new fluorogenic substrate for chymotrypsin. Analytical Biochemistry 70, 258-262. 